\title{
Variable Region Expression in the Antibody Responses of Infants Vaccinated with Haemophilus influenzae Type b Polysaccharide-Protein Conjugates \\ Description of a New $\lambda$ Light Chain-associated Idiotype and the Relation between Idiotype Expression, Avidity, and Vaccine Formulation
}

\author{
Dan M. Granoff, “ Penelope G. Shackelford, * Sandra J. Holmes, * \\ The Collaborative Vaccine Study Group, and Alexander H. Lucas* \\ *Division of Infectious Diseases, Edward Mallinckrodt Department of Pediatrics, Washington University School of Medicine, St. Louis \\ Children's Hospital, St. Louis, Missouri 63110; and ${ }^{\ddagger}$ Children’s Hospital Oakland Research Institute, Oakland, California 94609
}

\begin{abstract}
Haemophilus influenzae b polysaccharide (Hib PS)-protein conjugate vaccines differ chemically and immunologically. To determine whether anti-Hib PS variable region expression might differ according to vaccine formulation, infants were vaccinated at 2, 4, and 6 mo of age with Hib PS coupled to either meningococcal outer membrane protein complex (Hib PSOMPC) or tetanus toxoid (Hib PS-T), or Hib PS oligomers coupled to a mutant diphtheria toxin (Oligo-CRM). Two antiHib PS idiotypes were measured in sera obtained after the third injection: HibId-1, expressed by anti-Hib PS antibodies having the $x$ II-A2 variable region, and HibId-2, a newly defined crossreactive idiotype associated with a subset of anti-Hib PS antibodies having $\lambda$ VII variable regions. HibId-1 was present in 33 , 68, and 64\% of infants given either Hib PS-OMPC, OligoCRM, or Hib PS-T, respectively $(P<0.001)$. The respective values for HibId-2 were 47, 18, and $10 \%(P=0.001)$. Subjects who were vaccinated with Hib PS-OMPC or Hib PS-T and who produced detectable HibId-1-positive antibody, had significantly higher mean antibody avidity than subjects who did not produce HibId-1 positive antibodies. In contrast, Oligo-CRM evoked high avidity anti-Hib PS antibodies, irrespective of the idiotypic profile. These findings indicate fundamental differences in both variable region content and antibody quality elicited by different Hib PS conjugate vaccines. (J. Clin. Invest. 1993. 91:788-796.) Key words: antibody functional activity • cross-reactive idiotype $\bullet$ antibody repertoire $\bullet$ vaccine
\end{abstract}

The Collaborative Vaccine Study Group consisted of the following individuals who enrolled children in the prospective immunogenecity trials and provided serum samples for our study: T. V. Murphy, University of Texas, Southwestern, Dallas; M. T. Osterholm and J. McHugh, Minnesota Health Department, Minneapolis; E. L. Anderson and R. B. Belshe, St. Louis University School of Medicine; J. Esbenshade, University of Utah, Salt Lake City; M. Blatter, Upper St. Claire, PA; and H. Keyserling, Atlanta, GA. K. P. Guito and C. K. Meschievitz, Connaught Laboratories, Inc., Swiftwater, PA, organized and supervised one of the multicenter studies.

Address correspondence to Alexander H. Lucas, Children's Hospital Oakland Research Institute, 747 52nd Street, Oakland, CA 94609.

Received for publication 12 December 1991 and in revised form 8 October 1992.

J. Clin. Invest.

(c) The American Society for Clinical Investigation, Inc.

0021-9738/93/03/07881/09 \$2.00

Volume 91, March 1993, 788-796

\section{Introduction}

Successful induction of antibody responses to the Haemophilus influenzae type b capsular polysaccharide (Hib PS) ${ }^{1}$ in infants requires the conjugation of protein to the Hib PS (1-3). A variety of conjugate vaccine formulations have been prepared that differ in polysaccharide size, protein carrier, and covalent linkage (3). These structural differences result in vaccines with different immunological properties. For example, the Hib PSouter membrane protein complex (Hib PS-OMPC) vaccine, which consists of high molecular weight Hib PS covalently coupled to Neisseria meningitidis outer membrane protein (4), stimulates serum anti-Hib PS antibody production after a single injection in infants as young as 2 mo of age, but stimulates only modest increases in serum antibody upon subsequent immunizations at 4 and 6 mo of age (2, 5-7). In contrast, a vaccine consisting of $\mathrm{Hib}$ PS oligomers coupled to nontoxic mutant diphtheria toxin (Oligo-CRM), does not elicit significant Hib PS-specific antibody after a single injection at 2 mo of age (7), but primes for an anamnestic response as antibody production is stimulated upon two further immunizations at intervals of 1-2 mo (6-8). Another striking difference between these two conjugate vaccines is the requirement for carrier immunization. When Oligo-CRM is administered to infant rhesus monkeys without additional diphtheria toxoid (DT) carrier vaccination, anti-Hib PS antibody responses are poor, even after two or three injections (9). Similar results have been reported in human infants (10). However, immunization with Hib PS-OMPC, which has a carrier protein unrelated to DT, induces high antibody levels in the absence of DT vaccination $(9,10)$. Furthermore, a recent study shows that Oligo-CRM and Hib PS-tetanus toxoid (Hib PS-T) conjugate vaccines elicit antibodies of higher mean avidity than does the Hib PSOMPC conjugate (11). Taken together, these findings suggest that these different Hib PS conjugate vaccines elicit specific antibody responses by different mechanisms.

Previous data from a number of laboratories indicate that the human antibody repertoire to Hib PS is of limited structural diversity. IgG antibodies are restricted to the IgGl and IgG2 subclasses $(12,13), \kappa$ chain antibodies predominate over

1. Abbreviations used in this paper: CRI, cross-reactive idiotype; DT, diphtheria toxin; H, heavy; Hib PS, Haemophilus influenzae type b capsular polysaccharide; Hib PS-OMPC, Hib PS-outer membrane complex; Hib PS-T, Hib PS-tetanus toxoid; Id, idiotype; L, light; OligoCRM, Hib PS oligomers coupled to nontoxic mutant DT; RABA, radioantigen binding assay; $\mathrm{V}$, variable. 
$\lambda$ chain antibodies $(14,15)$, and a limited number of serum anti-Hib PS clonotypes are resolvable by isoelectric focusing $(12,16)$. Anti-Hib PS antibody heavy $(\mathrm{H})$ chain variable (V) regions are usually of the $\mathrm{VH} 3$ subgroup and are encoded by the VH 9.1 or VH 26 genes, or closely related genes (17-19). Anti-Hib PS VL regions may be $\kappa \mathrm{I}, \kappa \mathrm{II}, \kappa \mathrm{III}, \kappa \mathrm{IV}$ or $\lambda$, but the most commonly expressed $\mathrm{VL}$ region is derived from the $\mathrm{V}_{\kappa} \mathrm{II}$ gene, A2 (20-22). Further, a cross-reactive idiotype (CRI), designated HibId-1, is a marker for anti-Hib PS antibodies having the $\mathrm{V}_{\kappa}$ IIA2 light (L) chain (22), and HibId-1-positive antibodies comprise on the average more than half of the vaccineinduced serum anti-Hib PS induced in older children and adults (22-23).

With the exception of a few infants vaccinated with Hib PS-OMPC (23), no information is available on the expressed idiotypic/ $\mathrm{V}$ region antipolysaccharide repertoire in infants less than $7 \mathrm{mo}$ of age. Infants of this age are of particular interest for three reasons. First, they are the principal target population for conferring protection by vaccination with bacterial polysaccharides. Second, they are unresponsive to vaccination with unconjugated polysaccharides and the mechanism(s) of this anergy is unknown. Third, because of their young ages, the antibody repertoire expressed after vaccination of infants is unlikely to have been affected by prior environmental exposure to Hib PS.

The availability of Hib PS conjugate vaccines for use in infants 2-6 mo of age allows us to access the early antibody repertoire specific for a clinically relevant polysaccharide. In the present study, we have examined conjugate vaccine-induced antibodies of infants for expression of two idiotypes: HibId-1, and a newly defined CRI, HibId-2, which serves as a serological marker for a subset of $\mathrm{V}_{\lambda}$-containing anti-Hib PS antibodies. Our findings demonstrate that the relative expression of these two CRIs is dependent upon the conjugate vaccine formulation. In addition, we show that the anti-Hib PS antibody avidity differences elicited by vaccination with two of the conjugate vaccines correlate with the relative expression of these two idiotypes.

\section{Methods}

Subjects and vaccines. Sera from 189 healthy infants, who were vaccinated at 2, 4, and 6 mo of age with either Hib PS-OMPC (PedVAX$\mathrm{Hib}^{\mathrm{R}}$; Merck Sharp and Dohme, West Point, PA $)(n=43)$, Oligo-
CRM (HIBTiter ${ }^{\text {R }}$; Praxis Biologics, Rochester, NY) $(n=66)$, or Hib PS coupled to tetanus toxoid (ActHIB ${ }^{\text {TM}}$; Merieux Institute, Lyon, France) $(n=80)$, were available for this study. Blood was obtained immediately before and $1 \mathrm{mo}$ after the third dose of vaccine as part of ongoing comparative immunogenicity trials of various conjugate vaccines $(7,7 a)$, and serum anti-Hib PS antibody concentrations were determined. However, because of the small volumes of pre-third dose vaccination sera available, the additional studies described below were limited to the post-third dose vaccination samples. Selection of these sera was based on availability of sufficient quantity, and the presence of $\geq 0.8 \mu \mathrm{g} / \mathrm{ml}$ of anti-Hib PS antibody, the minimal concentration needed to perform the multiple immunologic assays on the small volumes of sera available.

The characteristics of the subjects and serum antibody concentrations are summarized in Table I. Group 1 received a standard dose consisting of $15 \mu \mathrm{g}$ of Hib PS and $256 \mu \mathrm{g}$ of protein, using a single lot of Hib PS-OMPC (lot 2381R). Group 2 received a standard dose of multiple commercial lots of Oligo-CRM, containing $10 \mu \mathrm{g}$ of $\mathrm{Hib}$ PS and 25 $\mu \mathrm{g}$ of protein. Group 3 received one of three investigational lots of $\mathrm{Hib}$ PS-T (lots S2141, S2181, and S2189), using a dose of $10 \mu \mathrm{g}$ of Hib PS and $20 \mu \mathrm{g}$ of protein. All vaccines were stored at $2^{\circ}-8^{\circ} \mathrm{C}^{\circ}$ and $0.5 \mathrm{ml}$ doses were administered intramuscularly in the thigh. Coded sera were stored at $-20^{\circ} \mathrm{C}$ in multiple vials. All assays were performed blindly without knowledge of the vaccine assignment.

Measurement of anti-Hib PS antibody. Total antibody to Hib PS was measured in a radioantigen binding assay (RABA) using ${ }^{125} \mathrm{I}$-labeled Hib PS as described previously (24). Antibody concentrations were determined by a standard curve generated using dilutions of the Office of Biologics reference serum pool. Anti-Hib PS antibody avidity was measured using a modified RABA, as described previously (25). Briefly, the avidity constant is determined by measuring the Hib PS binding capacity of a serum using two concentrations of ${ }^{125} \mathrm{I}-\mathrm{Hib} \mathrm{PS}$, $0.0025 \mathrm{nM}$ and $0.1 \mathrm{nM}$. The avidity constant is given by the relationship, $K=P /\{(1-P) \times[\mathrm{Ab}]\}$, where $K$ is the avidity constant, [Ab] is the concentration of anti-Hib PS antibody molecules (mol wt $=150,000$ ) determined from the RABA using $0.1 \mathrm{nM}^{125} \mathrm{I}-\mathrm{Hib} \mathrm{PS}$, and $P$ is the fraction of bound Hib PS molecules determined from the RABA using $0.0025 \mathrm{nM}^{125} \mathrm{I}$-Hib PS. $K$ is calculated from several serum dilutions having $P$ values between 20 and $80 \%$, and the final avidity value, in $\mathrm{nM}^{-1}$, was assigned based on the average.

Human monoclonal anti-Hib PS antibodies. The following human monoclonal anti-Hib PS antibodies were used in this study: LSF-2 $\left(\operatorname{IgA}_{1}, \lambda\right), \operatorname{ED} 8.4(\operatorname{IgM}, \kappa), \operatorname{RAY} 4\left(\operatorname{IgA}_{2}, \lambda\right), \operatorname{SB} 5 / D 6\left(\operatorname{IgA}_{1}, \lambda\right)$, ED6.1 $\left(\operatorname{IgG}_{2}, \kappa\right), \mathrm{JB} 21\left(\operatorname{IgG}_{2}, \lambda\right), \mathrm{JB} 32\left(\operatorname{lgA}_{1}, \lambda\right)$, ANN2 $(\operatorname{IgA}, \kappa)$, ANN23 $(\operatorname{IgM}, \kappa), \mathrm{CB} 6(\operatorname{IgA} 2, \lambda), \mathrm{CB} 1(\operatorname{IgA}, \kappa)$, and CA4 $(\operatorname{IgG} 2, \kappa)$. With the exception of CA-4, ANN2, ANN23, CB6, and CB1, the isolation, characterization, and sequence analyses of these $\mathrm{mAbs}$ are reported elsewhere $(18,26)$.

Table I. Characteristics of the Subjects

\begin{tabular}{|c|c|c|c|c|c|c|c|}
\hline \multirow[b]{2}{*}{$\begin{array}{l}\text { Subject } \\
\text { group }\end{array}$} & \multirow[b]{2}{*}{ Vaccine } & \multirow[b]{2}{*}{$\begin{array}{l}\text { No. } \\
\text { tested }\end{array}$} & \multirow[b]{2}{*}{$\begin{array}{c}\text { Age at third } \\
\text { vaccination } \\
\text { mean } \pm S D\end{array}$} & \multirow[b]{2}{*}{ White } & \multirow[b]{2}{*}{ Male } & \multicolumn{2}{|c|}{$\begin{array}{l}\text { Serum anti-Hib PS antibody* } \\
\text { geometric mean }\end{array}$} \\
\hline & & & & & & $\begin{array}{c}\text { Pre-third } \\
\text { vaccination }\end{array}$ & $\begin{array}{c}\text { Post-third } \\
\text { vaccination }\end{array}$ \\
\hline & & & months & $\%$ & $\%$ & \multicolumn{2}{|c|}{$\mu g / m l$} \\
\hline 1 & Hib PS-OMPC & 43 & $6.3 \pm 0.3$ & 98 & 47 & 8.2 & 10.3 \\
\hline 2 & Oligo-CRM & 66 & $6.3 \pm 0.5$ & 97 & 59 & 0.9 & 10.8 \\
\hline 3 & Hib PS-T & 80 & $6.4 \pm 0.5$ & 99 & 44 & 2.1 & 12.6 \\
\hline
\end{tabular}

\footnotetext{
* Subjects were vaccinated at 2, 4, and 6 mo of age as part of previous studies $(7,7 a)$. Anti-Hib PS antibody was measured by RABA in sera obtained immediately before the third injection and $1 \mathrm{mo}$ later. Samples were selected for analysis based on availability and the presence of $\geq 0.8 \mu \mathrm{g} / \mathrm{ml}$ in the post-third vaccination samples, the minimum concentration required for the different studies ( $<10 \%$ of each group were excluded).
} 
Production of $m A$ a antiidiotypic antibody ( $L u C 10)$ to $M A b$ LSF-2. $\mathrm{BALB} / \mathrm{c}$ mice were immunized in several subcutaneous sites with human mAb LSF-2 emulsified in CFA. A single dose consisted of $20 \mu \mathrm{g}$ of purified antibody. $3 \mathrm{wk}$ later the mice were immunized subcutaneously with the same dose of LSF- 2 in incomplete adjuvant. 5 wk later a third injection of $20 \mu \mathrm{g}$ of LSF-2 was administered intraperitoneally, and the spleen was taken $3 \mathrm{~d}$ later and used for hybridoma production. Hybridomas were prepared using established methods (27), by fusing spleen cells to the X63-Ag8.653 cell line (28) with $50 \%$ polyethylene glycol. A solid phase radioimmune assay was used to test hybridoma supernatants for antiidiotypic activity. This assay was essentially the same as that described in reference (22), except that microtiter wells were coated with purified LSF-2 mAb instead of purified serum anti-Hib PS antibody. Culture supernatants were screened for anti-LSF-2 idiotypic activity by measuring the ability of the supernatant to inhibit the binding of ${ }^{125}$ I-Hib PS to the solid phase adsorbed LSF-2 antibody. After subcloning by limiting dilution, a hybridoma clone was isolated that secreted a $\mathrm{mAb}(\operatorname{IgG} 1, \kappa)$, designated $\mathrm{LuC10}$, that completely inhibited the binding of Hib PS to MAb LSF-2. MAb LuC10 was isolated from ascites fluid by ammonium sulfate fractionation and ion exchange chromatography. Alkaline phosphatase was coupled to LuC10 using glutaraldehyde.

Characterization of $m A b L u C 10$. A previously described ELISA was used to assess the binding of LuC10 to human Ig (23). Briefly, wells of microtiter plates were coated with either human anti-Hib PS mAbs, human myeloma proteins (provided by Dr. Hans Spiegelberg, University of California, San Diego), or Hib PS-absorbed pooled intravenous gamma globulin. Wells were blocked with albumin, washed, and then reacted with varying concentrations of LuC10-alkaline phosphatase. After several hours of incubation, bound $\mathrm{LuCl} 0$ was detected with $p$-nitrophenyl phosphate solution.

The ability of Hib PS to inhibit the reaction between $\mathrm{LuC10}$ and LSF-2 was measured in ELISA (23). Solid phase adsorbed LSF-2 was reacted with varying concentrations of $\mathrm{Hib} \mathrm{PS}$, followed by addition of LuC10-alkaline phosphatase. Bound $\mathrm{LuCl} 0$ was detected using appropriate substrate, and optical density determined after $\sim 30 \mathrm{~min}$. The percent inhibition of $\mathrm{LuC10}$ binding by Hib PS was calculated as previously described (22).

The reaction between $\mathrm{mAb} \mathrm{LuCl} 10$ and human Ig was also examined by ELISA. Microtiter wells were coated overnight with LuC10 ( 10 $\mu \mathrm{g} / \mathrm{ml}$ PBS), blocked with albumin, and then reacted with postvaccination serum samples that had been absorbed with either Hib PS-Sepharose or unmodified Sepharose. The wells were washed and alkaline phosphatase conjugated antiisotypic antibodies were added for $3 \mathrm{~h}$. After washing, substrate solution was added and absorbance at $405 \mathrm{~nm}$ was measured after $\sim 30 \mathrm{~min}$.
Assays of anti-Hib PS HibId-1 and HibId-2 expression by LuC9 and LuC1O inhibition of Hib PS binding. HibId-1 and HibId-2 expression by total serum anti-Hib PS antibodies was quantified by measuring the degree to which the respective monoclonal antiidiotypic antibody inhibited the binding of ${ }^{125}$ I-Hib PS in the RABA. This assay has been described previously in detail $(22,23)$. Briefly, a dilution of serum or anti-Hib PS mAb, equivalent to an anti-Hib PS concentration between 0.8 and $2.0 \mu \mathrm{g} / \mathrm{ml}$, was mixed with an equal volume of either LuC9 $(60 \mu \mathrm{g} / \mathrm{ml}), \mathrm{LuCl} 0(60 \mu \mathrm{g} / \mathrm{ml})$, or diluent. After incubation, the Hib PS binding activity was determined by addition of $\sim 0.1 \mathrm{nM}$ ${ }^{125} \mathrm{I}$-Hib PS, followed by addition of $50 \%$ saturated ammonium sulfate. Inhibition of ${ }^{125} \mathrm{I}-\mathrm{Hib}$ PS binding by the anti-Id was determined as described previously (23). An ELISA was used to measure CRI expression by $\kappa$ and $\lambda$ anti-Hib PS antibodies. Postvaccination sera were screened for $\kappa$ and $\lambda$ anti-Hib PS antibodies using microtiter plates coated with Hib PS-poly-L-lysine and alkaline phosphatase coupled goat antibodies specific for human $\kappa$ or $\lambda$ chains (Tago Inc., Burlingame, CA ). 46 sera were selected for idiotypic analysis based on suffcient quantity, and the presence of $\geq 2.0 \mu \mathrm{g} / \mathrm{ml}$ of $\kappa$ - and $/$ or $\lambda$-specific anti-Hib PS antibody. 19 of these sera had sufficient $\kappa$ and $\lambda$ anti-Hib PS antibody to measure CRI expression in relation to each $\mathrm{L}$ chain; an additional 16 had sufficient $\kappa$ antibody but not $\lambda$, and 11 had sufficient $\lambda$ but not $\kappa$ antibody. This assay, which has been described previously for measurement of IgG1 and IgG2-specific CRI (23), entails incubating dilutions of human sera with a fixed concentration of LuC9 or $\mathrm{LuC10}$, and then transferring these sera to microtiter wells coated with Hib PS-poly-L-lysine. Bound antibody was detected with alkaline phosphatase coupled goat antibodies specific for either human $\kappa$ or $\lambda$ chains (Tago, Inc.), and inhibition of Hib PS binding by the anti-Id was calculated as previously described (23).

\section{Results}

$m A b$ LuC10 reacts with a combining site-associated idiotypic determinant of human $m A b L S F-2$. As described in Methods, a murine $\mathrm{mAb}$ designated $\mathrm{LuCl}(\mathrm{IgG} 1, \kappa)$ was isolated that inhibited the binding of human mAb LSF-2 $(\operatorname{IgA}, \lambda)$ to ${ }^{125} \mathrm{I}$ Hib PS in a solid phase radioimmune assay. Fig. 1 shows the dose-dependent inhibition of ${ }^{125} \mathrm{I}$-Hib PS binding by $\mathrm{LuC10}$ in a conventional liquid phase RABA. For comparison, previously published inhibition data with $\mathrm{mAb} \mathrm{LuC} 9$ are also shown (22). In this assay a fixed concentration $(0.2 \mu \mathrm{g} / \mathrm{ml})$ of antiHib PS mAbs LSF-2 or ED8.4, was reacted with varying concentrations of $\mathrm{LuC1} 10$ or LuC9, followed by addition of radiola-
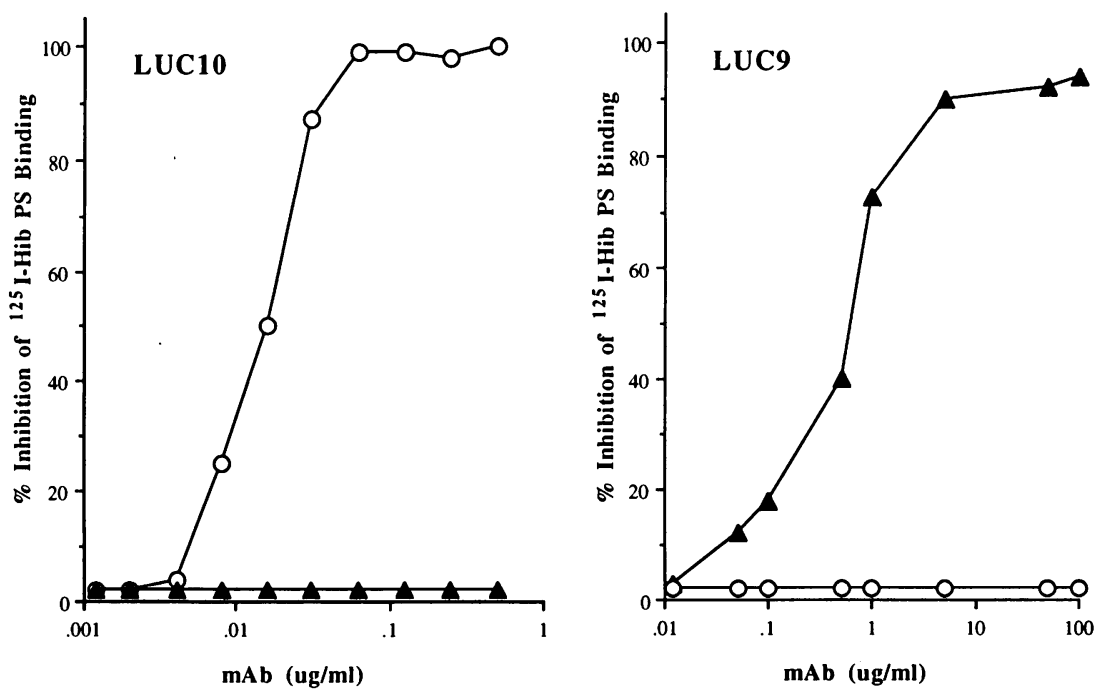

Figure 1. $\mathrm{mAb} \mathrm{LuCl} 10$ and $\mathrm{mAb} \mathrm{LuC} 9$ inhibition of ${ }^{125}$ I-Hib PS binding by human mAbs LSF-2 $(\circ)$ and ED8.4 ( $\triangle$ ) as determined in the radioantigen binding assay. A fixed concentration $(0.2$ $\mu \mathrm{g} / \mathrm{ml}$ ) of anti-Hib PS mAbs, LSF-2 or ED8.4, was reacted with varying concentrations of anti-Ids, Luc10 or Luc9 (x-axis). 
beled Hib PS and determination of binding activity. LuC10 inhibited $100 \%$ of LSF-2 Hib PS binding activity at concentrations as low as $0.1 \mu \mathrm{g} / \mathrm{ml}$. In contrast, no inhibition of ED8.4 ( IgM, $\kappa$ ) Hib PS binding was observed. ED8.4 is a human $\mathrm{mAb}$ specific for Hib PS and expresses the CRI, HibId-1, associated with $\kappa \mathrm{L}$ chains having the $\mathrm{A} 2 \mathrm{~V}$ region (22). With $\mathrm{LuC9}$, the reverse pattern was observed: > 90\% inhibition of ED8.4 Hib PS binding but no inhibition of LSF-2 Hib PS binding. Considerably higher concentrations of $\mathrm{LuC} 9$ were needed to inhibit ED8.4 compared to that of LuC10 needed to inhibit LSF-2.

The specificity of LuC10 was examined by direct ELISA, where LuC10-alkaline phosphatase was incubated in microtiter wells that had been coated with either LSF-2, ED8.4, human myeloma proteins (representative of the $\operatorname{IgM}, \operatorname{IgG}_{1,2,3,4}$, $\operatorname{Ig} A_{1}$, and $\operatorname{IgD} H$ chain isotypes, and $\kappa$ and $\lambda \mathrm{L}$ chain types), or pooled normal $\gamma$-globulin absorbed with Hib PS. LuC10 bound only to wells coated with LSF-2 (data not shown). Fig. 2 shows that the binding of $\mathrm{LuC10}$ to solid phase adsorbed LSF-2 was inhibited $90 \%$ by $10 \mu \mathrm{g} / \mathrm{ml} \mathrm{Hib} \mathrm{PS}$, and that $50 \%$ inhibition was achieved between .01 and $0.1 \mu \mathrm{g} / \mathrm{ml}$ of Hib PS.

Collectively, these data indicate that $\mathrm{mAb} \mathrm{LuC10}$ reacts with an idiotope (Id) on human mAb LSF-2 that is associated with the Hib PS-binding site, and that is distinct from the previously described major CRI, HibId-1. Hereafter we will refer to this new Id as HibId-2.

HibId-2 is a cross-reactive idiotype associated with antiHib PS antibodies having $\lambda$ L chains. HibId-2 is not a private Id restricted to mAb LSF-2, but is expressed by heterologous antiHib PS antibodies having only $\lambda L$ chains. This finding was demonstrated in an ELISA where $\mathrm{LuC10}$ was tested for its ability to inhibit the binding of either $k$ or $\lambda$ serum antibodies to Hib PS evoked by vaccination of infants with different conjugate vaccines. In this assay, $>20 \%$ inhibition is considered to be positive for idiotype expression ( $>3 \mathrm{SD}$ above background inhibition). As shown in Fig. $3 \mathrm{~A}$, LuC10 variably inhibited only $\lambda$ anti-Hib PS antibodies. This result implies that a subset of $\lambda$-bearing serum anti-Hib PS antibodies express HibId-2. In contrast, as previously described (22), Hibld-1 (as defined by LuC9 inhibition of Hib PS binding) was confined to a subset of anti-Hib PS serum antibodies having $\kappa$ L chains (Fig. $3 B$ ).

Further evidence that HibId-2 was restricted to $\lambda$-bearing anti-Hib PS antibodies was obtained using an ELISA where wells coated with $\mathrm{mAb} \mathrm{LuC10}$ were reacted with six postvaccination sera that had been absorbed with either Hib PS-Sepharose or as a negative control, unmodified Sepharose. The

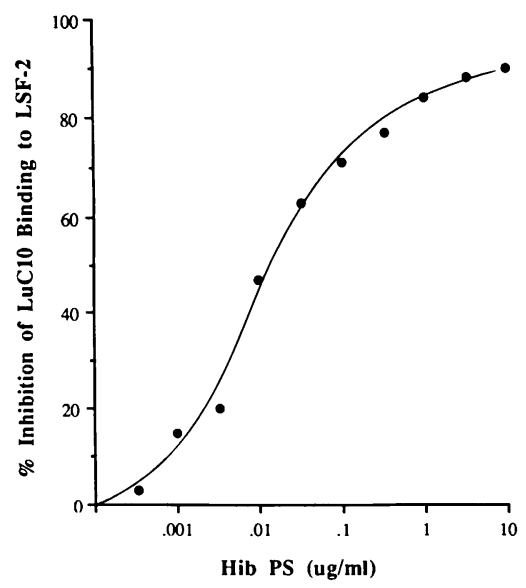

Figure 2. Hib PS inhibition of the reaction between $\mathrm{mAb} \mathrm{LuCl} 10$ and human mAb LSF-2. Microtiter wells, coated with mAb LSF-2, were reacted with alkaline phosphatase-coupled LuC10 in the absence or presence of various doses of Hib PS, and the percent inhibition was determined as described (22).
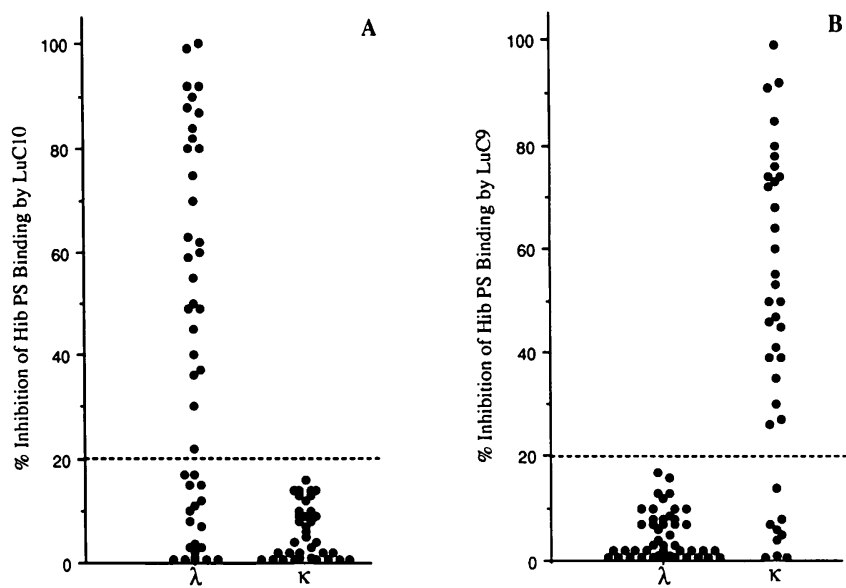

Figure 3. Light chain restriction of cross-reactive idiotypes. Percent inhibition of $\kappa$ or $\lambda$ anti-Hib PS binding by $\operatorname{LuC10}(A)$ or LuC9 $(B)$ was determined by ELISA as described in Methods. Postvaccination sera from infants immunized with different Hib PS conjugates (Table I) were selected based on the presence of $\geq 2.0 \mu \mathrm{g} / \mathrm{ml}$ of $\kappa$ and $/$ or $\lambda$-specific anti-Hib PS antibody as determined in ELISA.

LuC10-reactive Ig was detected with enzyme-conjugated antibodies specific for $\kappa$ or $\lambda \mathrm{L}$ chains or IgG, IgA, or IgM. The results obtained with two serum samples are shown in Fig. 4. MAb LuC10 reacted exclusively with $\lambda$ Ig and this reactivity was removed by absorption with Hib PS-Sepharose. In addition, the $\mathrm{LuCl}$-reactive Ig of these infant sera was of the IgG and IgM classes, with no detectable IgA. This isotype pattern reflects the isotype distribution of total anti-Hib PS antibodies in these samples as determined by ELISA using Hib PS coated wells, i.e., predominantly IgG with some IgM and little or no IgA (data not shown). The same pattern of idiotype and isotype reactivity was observed with the other four serum samples. Thus, HibId- 2 is restricted to $\lambda$ anti-Hib PS antibodies, and is expressed by IgG or IgM antibodies.

$m A b L u C 10$ reactivity with sequence-defined human $m A b s$ indicates that HibId-2 is a marker for a VגVII subset of antiHib PS antibodies. This L chain reactivity pattern was also seen with LuC10 inhibition of Hib PS binding by $\lambda$ chain bearing human mAbs. In addition to LSF-2, the Hib PS-specific mAbs SB5/D6 $\left(\operatorname{IgA} A_{1}, \lambda\right)$ and RAY $4\left(\operatorname{IgA} A_{2}, \lambda\right)$ were inhibited 65 and $100 \%$, respectively, by LuC10 when tested in the RABA inhibition assay. However, the Hib PS binding of three other mAbs also having $\lambda$ L chains, JB21, JB32, and CB6, was not inhibited by Luc10. Also, in keeping with the lack of $\mathrm{LuC10}$ inhibition of serum $\kappa$ antibodies in ELISA (Fig. 3 ) and the lack of inhibition of mAb ED8.4 (Fig. 1), Luc10 did not inhibit Hib PS binding of five other human $\kappa$ chain containing mAbs: ED6.1, CB1, ANN23, ANN2, and CA4, representative of antibodies having $\kappa \mathrm{I}, \kappa \mathrm{II}$, or $\kappa \mathrm{III}$ L chains ( reference 26 , and authors' unpublished observations). Thus, consistent with the $\mathrm{L}$ chain restriction observed with the serum antibodies, these inhibition data with the human mAbs indicate that HibId-2 is a CRI expressed by a subset of $\lambda$ anti-Hib PS antibodies.

The nucleotide sequences of the $\mathrm{VH}$ and $\mathrm{VL}$ regions have been determined for five of the six anti-Hib PS mAbs having $\lambda$ chains, including the three expressing HibId-2 $(18,26)$. All five antibodies use VH segments that are $93-98 \%$ homologous to the candidate germline gene 9.1 ( 18 , and authors' unpublished 

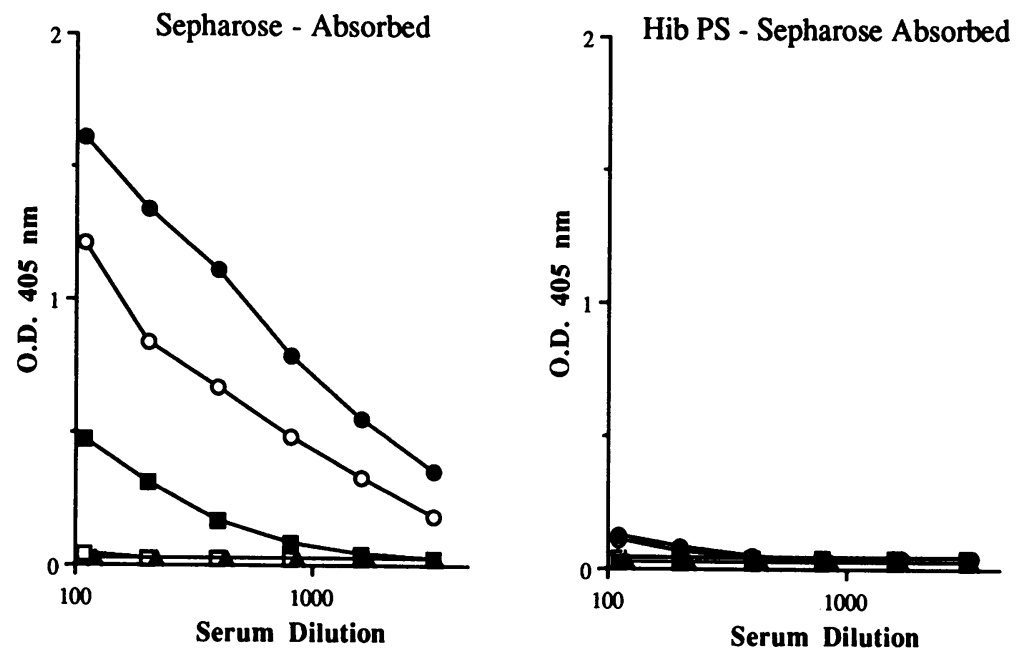

Serum 29790
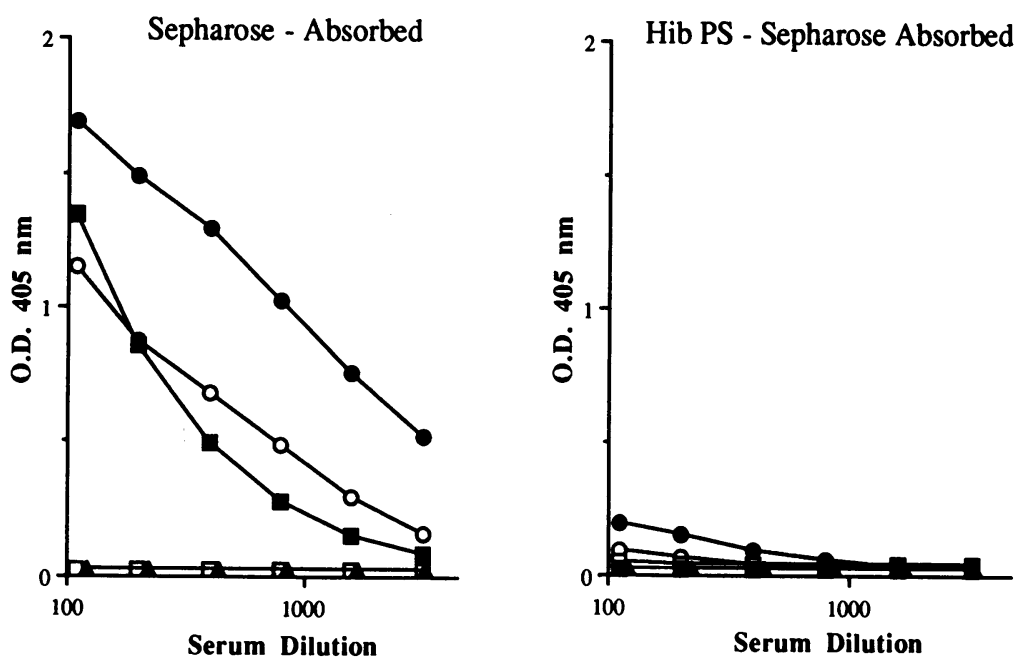

Figure 4. MAb LuC10 reacts with IgG and IgM anti-Hib PS antibodies having $\lambda \mathrm{L}$ chains. Two sera (29039 and 29790) obtained 1 mo after the third vaccination with Hib PS-OMPC, were absorbed with either Hib PS-Sepharose or unmodified Sepharose. Serial dilutions of the absorbed sera were then incubated in microtiter wells coated with $\mathrm{LuC10}$. LuC10-reactive Ig was detected using enzyme-conjugated antibodies specific for IgG (O), IgM $(\bullet), \operatorname{IgA}(\square), \kappa(\Delta)$, or $\lambda(\bullet)$.

observations). One mAb, JB21, is encoded by a V $\lambda$ II gene family member, and this antibody was negative for HibId-2. The remaining four antibodies use V $\lambda$ VII segments that are 96-98\% homologous to each other and that may originate from a single germline gene (26). Three of these four antibodies, LSF2, RAY4, and SB5/D6, were positive for HibId-2, and the fourth antibody JB32, was negative. The V $\lambda$ amino acid sequences of RAY4 and SB5 / D6 are 100 and 98\% homologous in the framework regions with LSF-2 (the immunogen used to prepare the anti-Id), and they each differed from LSF-2 by only three amino acid residues in the complementarity determining residues (CDRs) (26). For comparison, JB32, the Hibld-2-negative $\mathrm{mAb}$, is $98 \%$ homologous to LSF-2 in the framework regions, and differs from LSF-2 in eight CDR amino acid residues (26). Whether these amino acid differences account for the differences in HibId-2 expression has yet to be determined. Nonetheless, the data indicate that HibId-2 serves as a serologic marker for a subset of V $\lambda$ VII anti-Hib PS antibodies.

Different Hib PS conjugate vaccines induce different idiotypic expression after vaccination at 2, 4, and 6 mo of age. To examine the effect of vaccine formulation on idiotypic expres- sion, infants were immunized with either Hib PS-OMPC, Oligo-CRM, or Hib PS-T at 2, 4, and 6 mo of age, and HibId-1 and HibId-2 were measured in sera obtained $\sim 1$ mo after the third injection (Table II). A serum was considered positive for idiotype if $>20 \%$ of ${ }^{125} \mathrm{I}$-Hib PS binding was inhibited by the respective $\mathrm{mAb}$ anti-Id. A highly significant difference was observed in the frequency of expression of the two idiotypes among. the recipients of the different vaccines. HibId-1 was detected in $33 \%$ of the Hib PS-OMPC group, as compared with 68 and $64 \%$ of infants immunized with either Oligo-CRM or Hib PS-T, respectively $(P=0.001)$. The reverse pattern was seen with HibId-2; $47 \%$ of the Hib PS-OMPC-immunized subjects expressed HibId-2, whereas 18 and $10 \%$ of subjects vaccinated with either Oligo-CRM or Hib PS-T were positive for Hib Id-2, respectively $(P=0.001)$. Thus, significant differences are observed in the frequency of HibId-1 and HibId-2 expression in infants immunized with different vaccine formulations at 2, 4, and 6 mo of age. However, among subjects positive for HibId-1 or Hibld-2, the respective mean idiotype levels were not significantly different among the different vaccine groups, and on average, HibId-1 or HibId-2 accounted for $\sim 50 \%$ of the total serum anti-Hib PS antibody. 
Table II. Effect of Vaccine Formulation on CRI Expression after Vaccination of Infants at 2, 4, and 6 Mo of Age

\begin{tabular}{|c|c|c|c|c|c|}
\hline \multirow[b]{3}{*}{$\begin{array}{l}\text { Subject } \\
\text { group }\end{array}$} & \multirow[b]{3}{*}{ Vaccine } & \multicolumn{4}{|c|}{ Serum cross-reactive idiotype expression* } \\
\hline & & \multicolumn{2}{|c|}{ Hibld-1 } & \multicolumn{2}{|c|}{ Hibld-2 } \\
\hline & & No. positive $\mathrm{f}^{\ddagger}$ total & $\begin{array}{l}\text { Percent inhibition } \\
\text { by LuC9 } \\
\text { mean } \pm \mathrm{SD}^{s}\end{array}$ & No. positive $/$ total & $\begin{array}{l}\text { Percent inhibition } \\
\text { by } \mathrm{LuCl0} \\
\text { mean } \pm \mathrm{SD}^{\xi}\end{array}$ \\
\hline & & $\%$ & & $\%$ & \\
\hline 1 & Hib PS-OMPC & $14 / 43(33)^{\prime \prime}$ & $63 \pm 22$ & $20 / 43(47)^{9}$ & $47 \pm 17$ \\
\hline 2 & Oligo-CRM & $45 / 66(68)^{\prime \prime}$ & $55 \pm 22$ & $12 / 66(18)^{1}$ & $44 \pm 22$ \\
\hline 3 & Hib PS-T & $51 / 80(64)^{11}$ & $53 \pm 21$ & $8 / 80(10)^{9}$ & $43 \pm 12$ \\
\hline
\end{tabular}

* Serum was obtained 1 mo after the third injection. HibId-1 and HibId-2 were measured using RABA inhibition assay with mAbs LuC9 and LuC10, respectively. ${ }^{\ddagger}$ A serum was considered positive for Hibld-1 or Hibld-2 if $>20 \%$ of the Hib PS binding activity was inhibited by the respective mAb. ${ }^{\S}$ Of subjects positive for Hibld-1 or HibId-2. " $\chi^{2}=15 ; \mathrm{df}=2 ; P<0.001$. " $\chi^{2}=22$; $\mathrm{df}=2 ; P<0.001$.

Irrespective of vaccine formulation, both idiotypes were infrequently expressed in the same individual (Table III). Thus, the effect of vaccine formulation on HibId-2 expression is seen principally in subjects who did not express HibId-1. Among the HibId-1 negative subjects, HibId-2 was detected in $66 \%$ of those given Hib PS-OMPC vaccine (Group 1) as compared with $29 \%$ and $17 \%$ of children given Oligo-CRM or Hib PS-T (Groups 2 and 3), respectively $(P<0.001)$.

Associations between idiotype expression and anti-Hib PS antibody avidity. Recently we reported that the different Hib PS conjugate vaccines elicit serum antibodies of different average avidities (11). To determine whether idiotype expression might be correlated with these differences in avidity, we stratified the previously determined antibody avidity values by vaccine group and idiotypic profile (Table IV). By measuring two idiotypes, we can stratify the vaccinees into four categories based upon HibId-1/HibId-2 expression: $+/+,+1-,-1+$, and $-/-$. Since overall only $5 \%$ of subjects were positive for both idiotypes $(+/+)$, there were too few subjects in this group for statistical analysis. We therefore restricted our comparisons

Table III. Relation Between HibId-1 and HibId-2 Expression in Infants Immunized with Different Hib PS Conjugate Vaccine Formulations

\begin{tabular}{cccccc}
\hline $\begin{array}{c}\text { Subject } \\
\text { group }\end{array}$ & Vaccine & Hibld-1* & $\begin{array}{c}\text { No. of } \\
\text { subjects }\end{array}$ & $\begin{array}{c}\text { No. of } \\
\text { subjects } \\
\text { positive } \\
\text { for Hibld-2 }\end{array}$ & $\begin{array}{c}\text { Percent } \\
\text { inhibition } \\
\text { by LuC10 } \\
\text { mean } \pm \text { SD }^{\ddagger}\end{array}$ \\
\hline & & & & $\%$ & \\
1 & Hib PS-OMPC & + & 14 & $1(7)$ & 46 \\
& & - & 29 & $19(66)^{\S}$ & $47 \pm 17$ \\
2 & Oligo-CRM & + & 45 & $6(13)$ & $36 \pm 19$ \\
3 & Hib PS-T & - & 21 & $6(29)^{\S}$ & $51 \pm 25$ \\
& & + & 51 & $3(6)$ & $35 \pm 10$ \\
& & - & 29 & $5(17)^{\S}$ & $48 \pm 11$ \\
\hline
\end{tabular}

Subjects were vaccinated at 2, 4, and 6 mo of age. HibId- 1 and Hibld-2 were measured in sera obtained 1 mo after the third injection. ${ }^{*}$ Criterion for Hibld-1 and Hibld-2 positivity is indicated in legend to Table II. ${ }^{\ddagger}$ Of subjects positive for HibId-2. ${ }^{8} \chi^{2}=15$; df $=2 ; P<0.001$. to the other three idiotypic categories. Among infants given three injections of Hib PS-OMPC vaccine, those who were HibId $-1^{+} /$HibId $-2^{-}$had significantly higher mean antibody avidity than those who were either HibId-1 ${ }^{-} /$Hibld- $2^{+}$or Hibld$1^{-} /$Hibld-2- $(P<0.001)$. In contrast, when the same analysis was performed for the infants given three injections of OligoCRM vaccine, there were no significant differences in the respective mean avidities of the groups with different idiotypic profiles $(P>0.27)$. Further, in all three idiotypic categories analyzed, infants given Oligo-CRM showed higher mean avidity than children with the respective idiotypic categories given Hib PS-OMPC $(P \leq 0.05)$. Previously we found that when analyzed as a group, infants given Oligo-CRM had higher mean avidity than those vaccinated with either Hib PS-OMPC or Hib PS-T (11). The present analysis indicates that this difference between the mean avidity of antibodies elicited by Oligo-CRM and Hib PS-T can be accounted for by the lower avidity antibodies in the Hib PS-T-vaccinated children who were negative for HibId-1 $(P<0.001)$. In contrast, the majority of infants responded to Hib PS-T vaccination by producing HibId- 1 antibodies, and these subjects had nearly identical mean antibody avidity as the corresponding infants given Oligo-CRM (2.55 $\mathrm{nM}^{-1}$ vs. $2.73 \mathrm{nM}^{-1}, P=0.6$ ).

\section{Discussion}

Molecular characterization of clonally purified serum anti-Hib PS antibodies $(17,20,21)$, and nucleic acid sequence analyses $(18,26)$ indicate that the antibody response to the Hib PS antigen is quite restricted with respect to $\mathrm{V}$ region gene utilization. The $\mathrm{H}$ chains generally use genes homologous to one of two germline sequences from the VHIII family (18-21), and the $\mathrm{L}$ chains are characterized by selective expression of a limited number of $\kappa$ and $\lambda V$ region genes (19-22). Sequencing of antibody $\mathrm{V}$ genes provides important information on the potential genetic repertoire of an immune response, and on the possible relationship between antibody function and structure. However, this approach is not practical for investigation of factors such as vaccine formulation, that may influence the expressed immune repertoire of populations. To address this question, we have employed anti-Id reagents of defined specificity to extend our previous analysis of the idiotype expression of serum antibodies evoked by different Hib PS vaccines. Previ- 
Table IV. Anti-Hib PS Idiotype and Avidity after Immunization with Conjugate Vaccine

\begin{tabular}{|c|c|c|c|c|c|c|}
\hline \multirow[b]{2}{*}{ Hibld-1/Hibld-2 } & \multicolumn{2}{|c|}{ Hib PS-OMPC } & \multicolumn{2}{|c|}{ Oligo-CRM } & \multicolumn{2}{|c|}{ Hib PS-T } \\
\hline & No. & $\begin{array}{c}\text { Avidity } \\
\text { mean } \pm \text { s.e., } \\
\mathrm{nM}^{-1}\end{array}$ & No. & $\begin{array}{c}\text { Avidity } \\
\text { mean } \pm \text { s.e. } \\
\mathrm{nM}^{-1}\end{array}$ & No. & $\begin{array}{c}\text { Avidity } \\
\text { mean } \pm \text { s.e., } \\
\mathrm{nM}^{-1}\end{array}$ \\
\hline$+1-$ & 10 & $1.76 \pm 0.16^{\mathrm{a} *}$ & 20 & $2.73 \pm 0.31^{\mathrm{d}}$ & 22 & $2.55 \pm 0.24^{8}$ \\
\hline$-1+$ & 16 & $0.84 \pm 0.12^{b}$ & 4 & $1.89 \pm 0.69^{e}$ & 1 & 1.07 \\
\hline$-1-$ & 10 & $0.94 \pm 0.16^{c}$ & 9 & $2.79 \pm 0.46^{\mathrm{f}}$ & 13 & $1.26 \pm 0.31^{\mathrm{h}}$ \\
\hline$+/+$ & 1 & 2.23 & 1 & 1.71 & 2 & $1.52 \pm 0.14$ \\
\hline
\end{tabular}

Infants were immunized at 2,4 , and 6 mo of age. Avidity and idiotype expression were measured in sera obtained $\sim 1$ mo after the third injection. Of the 189 infants in the present study, avidity had been previously measured in sera on 109 of these subjects as part of a separate study (11). Because of insufficient quantities of sera, the avidity and idiotype analyses were limited to these samples. ${ }^{*}$ Significance testing by analysis of variance: a vs. b, $P<0.001$; a vs. c, $P<0.001$; g vs. h, $P=0.002$. a vs. d, $P=0.05$; b vs. e, $P=0.003$; c vs. f, $P<0.001$; f vs. h, $P<0.001$.

ously, we found that antibodies to Hib PS frequently express HibId-1, a CRI which is localized to the VkIIA2 light chain (22). Indeed, HibId-1 is a highly prevalent CRI, and is detected in the sera of $\sim 80 \%$ of subjects vaccinated at 1.7 to $57 \mathrm{y}$ of age with Hib PS or Hib PS coupled to diphtheria toxoid. HibId-1 comprises on the average more than $50 \%$ of the serum antibody of children and adults $(22,23)$. However, most of the subjects in these studies had anti-Hib PS antibody in prevaccination sera, presumably as a result of natural exposure to Hib or other bacteria that elaborate polysaccharides cross-reactive with $\mathrm{Hib}$ PS (29). Therefore, the antibody repertoire expressed after vaccination may have been affected by prior environmental exposures.

In the present study, we examined the idiotypic expression of the antibody response to Hib conjugate vaccination of infants 2-6 mo of age in whom the effect of prior environmental exposures to cross-reactive polysaccharides would be expected to be minimized because of their young ages. We also examined the idiotypic repertoire induced in response to several structurally distinctive Hib conjugate vaccines that appear to elicit antibody responses by different immunologic mechanisms (30-32). Finally, we described a new CRI, HibId-2, that allowed us to extend our analysis to a subset of non-HibId-1 V regions expressed after vaccination. This new CRI, HibId-2, is defined by $\mathrm{mAb}$ LuC10. This anti-Id reacted specifically with anti-Hib PS antibodies having $\lambda \mathrm{L}$ chains. Further, comparison between Hibld-2 expression and $\mathrm{V} \lambda$ nucleotide sequence suggested that HibId-2 was confined to a subset of V $\lambda$ VII anti-Hib PS antibodies.

The two anti-Id reagents were used to investigate the effect of conjugate vaccine formulation on $\mathrm{V}$ region expression in postvaccination sera. After three injections beginning at 2 mo of age, Hib PS-OMPC evoked very different patterns of idiotypic expression than that observed after three injections of Oligo-CRM and Hib PS-T given at the same ages. With both Oligo-CRM and Hib PS-T, HibId-1 was more commonly expressed, and HibId-2 was less common, than after vaccination with Hib PS-OMPC vaccine at this age. Hibld-2 tended to be more frequently expressed after Oligo-CRM than Hib PS-T but this difference was not significant ( 18 vs. $10 \%, P \geq 0.15$ ). These data indicate that the anti-Hib PS VL regions elicited by Oligo-CRM or Hib PS-T vaccination, appear similar to each other, but different from those elicited by Hib PS-OMPC vaccination.
Of the three Hib conjugate vaccines used in our studies, Hib PS-OMPC is unique in that it is able to elicit a primary antibody response in 2-mo-old infants $(2,5-7)$, whereas beginning at this age, the other two conjugates require two or three injections to elicit antibody (6-8). This difference in immunogenicity between the three conjugate vaccines is shown by the significantly higher antibody levels before the third dose, in the infants who had received the OMPC conjugate than those who received the other two conjugates (Table I). Recently we observed that HibId-2 expression following a single injection of PRP-OMPC tends to diminish with age (33). Thus, the higher usage of HibId-2 after three injections of the Hib PS-OMPC conjugate observed in the present study, may relate to the unique ability of this conjugate to induce HibId-2 antibodies in 2-mo-old infants and thereby fix the idiotypic repertoire at this age. In contrast, the antibody response to the other two conjugates occurred at 4-6 mo of age, by which time the potential idiotypic repertoire may have shifted.

The mechanism(s) by which the Hib PS-OMPC vaccine is able to induce a primary antibody response in 2-mo-old infants, as well as induce such a distinctive idiotypic profile, is unknown. This conjugate uses an outer membrane complex from Neisseria meningiditis, and this carrier protein may uniquely modulate the infant's immune response. Recently, this conjugate vaccine was found to be mitogenic for murine $B$ cells (30-32) and, thus, in addition to having thymic dependent properties, the Hib PS-OMPC conjugate may have properties of a thymic-independent type 1 antigen. Conceivably, the differences in $\mathrm{V}$ region expression evoked by the different $\mathrm{Hib}$ conjugate vaccines may reflect differences in the relative contributions of thymic-dependent and thymic-independent mechanisms operative in the antibody responses to Oligo-CRM or Hib PS-T compared to those operating with the Hib PS-OMPC conjugate. Evidence in a murine antihapten antibody response suggests that different $B$ cell subpopulations are stimulated by TI and TD forms of hapten (34). Therefore, different Hib PS conjugate vaccines may stimulate distinctive B cell subsets, and these subsets could differ in their potential to express HibId-1 and/or HibId-2. The conjugate vaccines may also differ in their capacity to promote somatic mutation and this property could affect $\mathrm{V}$ repertoire content. For example, the lower frequency of expression of HibId-2 antibodies after vaccination with either the tetanus or Oligo-CRM conjugates as compared to OMPC, could result from the accumulation of mutations 
that extinguish the idiotope. In this case, lack of Hibld-2 expression would not reflect lack of utilization but rather somatic modification of the V $\lambda$ VII region. Consistent with this interpretation are the data with the anti-Hib PS mAbs, showing that HibId-2 is expressed by a subset (three of four) of antibodies using V $\lambda$ VII.

Recently we compared the avidities of serum anti-Hib PS antibodies evoked by vaccination with Oligo-CRM, Hib PSOMPC, or Hib PS-T conjugate at 2, 4, and 6 mo of age (11). Oligo-CRM stimulated antibodies of higher mean avidity than those elicited by Hib PS-OMPC vaccination, while the mean avidity of antibodies evoked by Hib PS-T was intermediate, being significantly higher than that of the Hib PS-OMPC group but lower than that of the group given Oligo-CRM. Further, there was an inverse correlation between the serum antibody concentration required to activate complement-mediated bacteriolysis of Hib, and the avidity of the anti-Hib PS antibody, a finding that suggests that differences in antibody avidity may play an important role in conferring protection against Hib disease. These differences in antibody avidity were likely a result of $\mathrm{V}$ region differences and not isotypic differences, since on average $>90 \%$ of the anti-Hib PS antibody responses to each of the conjugates was IgG and there was no correlation between the magnitude of the IgM response and avidity (11).

In the present study, we examined the relationship between average serum anti-Hib PS antibody avidity and CRI expression. The data indicate that both immunogen (vaccine formulation) and CRI content correlate with antibody avidity. Subjects who were immunized with either Hib PS-OMPC or Hib PS-T vaccines and who produced detectable HibId-1-positive antibody, had significantly higher mean avidity than subjects who did not produce HibId-1-positive antibodies. Although we have not measured avidity of clonally purified anti-Hib PS antibodies, this correlation suggests that antibodies expressing HibId-1, i.e., having the unmutated $\mathrm{V}_{\kappa} \mathrm{II}-\mathrm{A} 2$ region $(21,22,26)$, have higher avidity for Hib PS than the non-HibId-1 population induced by Hib PS-T or Hib PS-OMPC. This putative higher avidity of $V_{\kappa} I I A 2$ antibodies may confer a selective advantage in protection against $\mathrm{Hib}$ disease and thereby maintain the nonpolymorphic A2 gene in the population.

However, the pattern of average antibody avidity and CRI expression seen after Oligo-CRM vaccination suggests that non-HibId-1 antibodies are not necessarily of lower avidity. After three injections of Oligo-CRM, the mean antibody avidity in sera not containing HibId-1/ $\mathrm{V}_{K}$ IIA2 was similar to that of sera having HibId-1 antibodies. In addition, vaccination with Oligo-CRM appeared to induce uniformly higher avidity anti-Hib PS antibodies than either Hib PS-OMPC or Hib PS-T, irrespective of the idiotypic profile.

The induction of higher avidity antibodies by the OligoCRM vaccine may result from the presence of Hib PS oligomers in the Oligo-CRM conjugate rather than the higher molecular weight form of Hib PS that is present in the tetanus and OMPC conjugates. The oligomeric form of Hib PS may selectively activate B cell clones having high affinity receptors. In addition, the Oligo-CRM conjugate may elicit greater $\mathrm{T}$ cell help compared with the other two conjugates, which may favor greater affinity maturation of HibId-1-negative antibody populations by promoting more extensive somatic mutation during the secondary immune response. The Hib PS conjugate vaccines differ with respect to the type of carrier molecule, the nature of the chemical linkage between the polysaccharide and the protein, and molecular size. These factors could affect the immunological properties of the vaccines, such as clearance, processing, and retention in reticuloendothelial tissues and, thus, possibly contribute to both the observed avidity differences and the distinct idiotype profiles induced by the different conjugate vaccines.

In previous studies, differences in mean avidities of two- or threefold were associated with greater than sixfold differences in the ability of anti-Hib PS antibodies to activate complement-mediated bactericidal activity (11) and opsonic activity (35). Thus, vaccine formulations that evoke higher avidity antibody may offer greater protection against invasive Hib disease. There are clear differences in $\mathrm{V}$ region usage and in the mean avidities of the anti-Hib PS antibodies in sera of infants vaccinated with different conjugate vaccines. These factors may be important in the design of future vaccines aimed at optimizing protective immunity.

\section{Acknowledgments}

Dr. Y. Schlesinger performed some of the measurements of antibody avidity. Venita Boelloeni, Ann Grace, Anthony Quinn, and Marina Kitamura provided expert technical assistance. Drs. Elisabeth Adderson and William Carroll performed the sequence analysis of the hybridomas.

This work was supported by grants AI-17962, AI-21842, AI-19350, and AI 25008 from the National Institute of Allergy and Infectious Diseases, National Institutes of Health.

\section{References}

1. Insel, R. A., and P. W. Anderson. 1986. Oligosaccharide-protein conjugate vaccines induce and prime for oligoclonal IgG antibody responses to the Haemophilus influenzae b capsular polysaccharide in human infants. J. Exp. Med. 163:262-269.

2. Einhorn, M. S., G. A. Weinberg, E. L. Anderson, P. D. Granoff, and D. M. Granoff. 1986. Immunogenicity in infants of Haemophilus influenzae type b polysaccharide in a conjugate vaccine with Neisseria meningitidis outer-membrane protein. Lancet. ii:299-302.

3. Weinberg, G. A., and D. M. Granoff. 1988. Polysaccharide-protein conjugate vaccines for the prevention of Haemophilus influenzae type b disease. $J$. Pediatr. 113:621-631.

4. Marburg, S., D. Jorn, R. L. Tolman, B. Arison, J. McCauley, P. J. Kniskern, A. Hagopian, and P. P. Vella. 1986. Biomolecular chemistry of macromolecules: synthesis of bacterial polysaccharide conjugate with Neisseria meningitidis membrane protein. J. Am. Chem. Soc. 108:5282-5287.

5. Lenoir, A. A., P. D. Granoff, and D. M. Granoff. 1987. Immunogenicity of Haemophilus influenzae type $\mathrm{b}$ polysaccharide-Neisseria meningitidis outer membrane protein conjugate vaccine in 2- to 6-month old infants. Pediatrics. 80:283-287.

6. Decker, M. D., K. M. Edwards, R. Bradley, and P. Palmer. 1992. Comparative trial in infants of four conjugate Haemophilus influenzae type b vaccines. $J$. Pediatr. 120:184-189.

7. Granoff, D. M., E. L. Anderson, M. T. Osterholm, S. J. Holmes, J. E. McHugh, R. B. Belshe, F. Medley, and T. V. Murphy. 1992. Differences in the immunogenicity of three Haemophilus influenzae type b conjugate vaccines in infants. J. Pediatr. 121:187-194.

7a. Holmes, S. J., B. Fritzell, K. P. Guito, J. Esbenshade, M. M. Blatter, K. S. Reisinger, H. Keyserling, E. P. Rothstein, H. H. Bernstein, S. Feldman, and D. M. Granoff. 1993. The immunogenicity of Haemophilus influenzae type b polysaccharide-tetanus toxoid conjugate vaccine in infants. Am.J. Dis. Child. In Press.

8. Madore, D. V., C. L. Johnson, D. C. Phipps, Pennridge Pediatric Associates, L. A. Popjoy, R. Eby, and D. H. Smith. 1990. Safety and immunologic response to Haemophilus influenzae b oligosaccharide-CRM 197 conjugate vaccine in 1- to 6-month-old infants. Pediatrics. 85:331-337.

9. Vella, P. A., and R. W. Ellis. 1991. Immunogenicity of Haemophilus influenzae type b conjugate vaccines in infant rhesus monkeys. Pediatr. Res. 29:1013.

10. Granoff, D. M., M. H. Rathore, S. J. Holmes, P. D. Granoff, and A. H. Lucas. 1993. Effect of immunity to the carrier protein on antibody responses to Haemophilus influenzae type b conjugate vaccines. Vaccine. 11:S44-S49. 
11. Schlesinger, Y., D. M. Granoff, and the Vaccine Study Group. 1992. Avidity and bactericidal activity of anti-capsular antibodies elicited by different Haemophilus influenzae type b conjugate vaccines. J. Am. Med. Assoc. 267:1489-1494.

12. Shackelford, P. G., D. M. Granoff, S. J. Nelson, M. G. Scott, D. S. Smith, and M. H. Nahm. 1987. Subclass distribution of human antibodies to Haemophilus influenzae b polysaccharide. J. Immunol. 138:587-592.

13. Mäkelä, O., P. Mattila, N. Rautonene, I. Seppälä, J. Eskola, and H. Käyhty. 1987. Isotype concentrations of human antibodies to Haemophilus influenzae type b polysaccharide in young adults immunized with the polysaccharide as such or conjugated to a protein (Diphtheria toxoid). J. Immunol. 139:1999-2004.

14. Insel, R. A., P. Anderson, M. E. Pichichero, S. Schuster, M. D. Amstey, G. Ekborg, and D. H. Smith. 1982. Anticapsular antibody to Haemophilus influenzae type b. In Haemophilus influenzae, Epidemiology, Immunology, and Prevention of Disease. S. H. Sell and P. E. Wright, editors. Elsevier Science Publishing Co., Inc., New York. 155-168.

15. Ambrosino, D. M., V. Greif, C. Thompson, and G. R. Siber. 1990. $\kappa$ and $\lambda$ light chain composition of antibody to the capsular polysaccharide of Haemophilus influenzae type b. J. Infect. Dis. 161:922-925.

16. Insel, R. A., A. Kittelberger, and P. Anderson. 1985. Isoelectric focusing of human antibody to the Haemophilus influenzae b capsular polysaccharide: restricted and identical spectrotypes in adults. J. Immunol. 135:2810-2816.

17. Scott, M. G., J. J. Tarrand, D. L. Crimmins, D. W. McCourt, N. R. Siegel, C. E. Smith, and M. H. Nahm. 1989. Clonal characterization of the human IgG antibody repertoire to Haemophilus influenzae type b polysaccharide. II. IgG antibodies contain $\mathrm{VH}$ genes from a single family and VL genes from at least four VL families. J. Immunol. 143:293-298.

18. Adderson, E. A., P. G. Shackelford, A. Quinn, and W. L. Carroll. 1991. Restricted Ig $\mathrm{H}$ chain $\mathrm{V}$ gene usage in the human antibody response to Haemophilus influenzae type b capsular polysaccharide. J. Immunol. 147:1667-1674.

19. Silverman, G. J., and A. H. Lucas. 1991. Variable region diversity in human circulating antibodies specific for the capsular polysaccharide of Hae mophilus influenzae type b: preferential usage of two types of $\mathrm{VH} 3$ heavy chains. J. Clin. Invest. 88:911-920.

20. Scott, M. G., D. L. Crimmins, D. W. McCourt, G. Chung, K. F. Schable, R. Thiebe, E.-M. Quenzel, H. G. Zachau, and M. H. Nahm. 1991. Clonal characterization of the human IgG antibody repertoire to Haemophilus influenzae type b polysaccharide IV. The less frequently expressed VL are heterogeneous. $J$. Immunol. 147:4007-4013.

21: Scott, M. G., J. J. Tarrand, D. L. Crimmins, D. W. McCourt, I. Zocher, R. Thiebe, H. G. Zachau, and M. H. Nahm. 1989. Clonal characterization of the human antibody repertoire to Haemophilus influenzae b polysaccharide. III. A single VkIl gene and one of several Jk genes are joined by an invariant arginine to form the most common $L$ chain V region. J. Immunol. 143:4110-4116.

22. Lucas, A. H., R. J. Langley, D. M. Granoff, M. H. Nahm, M. Y. Kita- mura, and M. G. Scott. 1991. An idiotypic marker associated with a germ-line encoded $\kappa$ light chain variable region that predominates the vaccine-induced human antibody response to the Haemophilus influenzae b polysaccharide. $J$. Clin. Invest. 88:1811-1818.

23. Lucas, A. H., and D. M. Granoff. 1990. A major crossreactive idiotype associated with human antibodies to the Haemophilus influenzae b polysaccharide: expression in relation to age and immunoglobulin $\mathrm{G}$ subclass. J. Clin. Invest. 85:1158-1166.

24. Granoff, D. M., P. G. Shackelford, J. P. Pandey, and E. G. Boies. 1986. Antibody responses to Haemophilus influenzae type b polysaccharide vaccine in relation to $\mathrm{Km}(1)$ and $\mathrm{G} 2 \mathrm{~m}(23)$ immunoglobulin allotypes. J. Infect. Dis. 154:257-264.

25. Griswold, W. R., A. H. Lucas, J. F. Bastian, and G. Garcia. 1989. Functional affinity of antibody to the Haemophilus influenzae b polysaccharide. $J$. Infect. Dis. 159:1083-1087.

26. Adderson, E. A., P. G. Shackelford, R. A. Insel, A. Quinn, P. M. Wilson, and W. L. Carroll. 1992. Immunoglobulin light chain variable region gene sequences for human antibodies to Haemophilus influenzae type b capsular polysaccharide are dominated by a limited number of $\mathrm{V}_{\boldsymbol{K}}$ and $\mathrm{V} \lambda$ segments and $\mathrm{VJ}$ combinations. J. Clin. Invest. 89:729-738.

27. Fazekas de St. Groth, S., and D. Scheidegger. 1980. Production of monoclonal antibodies: strategies and tactics. J. Immunol. Methods. 47:129-144.

28. Kearney, J. F., A. Radbruch, B. Liesengang, and K. Rajewsky. 1979. A new mouse myeloma cell line that has lost Ig expression but permits construction of antibody-secreting hybrid cell lines. J. Immunol. 123:1548-1550.

29. Robbins, J. B., R. Schneerson, M. P. Glode, W. Vann, M. S. Shiffer, T.-Y. Liu, J. C. Parke, and C. Huntley. 1975. Cross-reactive antigens and immunity to diseases caused by encapsulated bacteria. J. Allergy Clin. Immunol. 56:141-151.

30. Donnelly, J. J., R. R. Deck, and M. A. Liu. 1990. Immunogenicity of Haemophilus influenzae $\mathrm{b}$ polysaccharide-Neisseria meningitidis outer membrane protein complex conjugate vaccine. J. Immunol. 145:3071-3079.

31. Stein, K. 1992. Thymus-independent and thymus-dependent responses to polysaccharide antigens. J. Infect. Dis. 165:S49-S52.

32. Liu, M. A., A. Friedman, A. I. Oliff, J. Thai, D. Martinez, R. R. Deck, J. C. T. Shieh, T. D. Jenkins, J. J. Donnelly, and L. A. Hawe. 1992. A vaccine carrier derived from Neisseria meningitidis with mitogenic activity for lymphocytes. Proc. Natl. Acad. Sci. USA. 89:4633-4637.

33. Lucas, A. H., F. H. Azmi, C. M. Mink, and D. M. Granoff. 1993. Age-dependent variable region expression in the human antibody response to the $\mathrm{Hae}$ mophilus influenzae type b polysaccharide. J. Immunol. In Press.

34. Tittle, T. V., and M. B. Rittenberg. 1978. Distinct subpopulations of IgG memory B cells respond to different molecular forms of the same hapten. $J$. Immunol. 121:936-941.

35. Amir, J., X. Liang, and D. M. Granoff. 1990. Variability in the functional activity of vaccine-induced antibody to Haemophilus influenzae type b. Pediatr. Res. 27:358-364. 